\title{
ADAMS-based Dynamics Simulation Analysis of Megawatt Wind Turbine Brakes
}

\author{
Zhihua Sha, Wei Liu, Yu Liu, Fujian Ma, Shengfang Zhang ${ }^{\mathrm{a}}$ \\ School of Mechanical Engineering, Dalian Jiao Tong University, Dalian, 116028, China \\ aemail: zsf@djtu.edu.com
}

Keywords: wind turbine brakes; rigid-flexible-coupling; dynamical simulation

\begin{abstract}
Since the wind turbine brake is not easy to replace and repair in the high-altitude operations, it requires high reliability and high braking performance. Accordingly, a dynamic model is established for the megawatt wind turbine brake. By simulating and analyzing the braking process, the braking curves and contact force curves of typical conditions are derived with the considering of the friction coefficient, braking power and flexible members. This approach provides a model reference for structural design and optimization of megawatt wind turbine brake.
\end{abstract}

\section{Introduction}

Because the wind energy is inexhaustible and renewable without transportation and pollution, wind turbine has become a method of generate electricity with the most mature technology and the most commercial prospects in the field of new energy [1] [2]. Wind turbine brakes are essential supporting equipments in the process of wind turbine generation. The research and development of wind turbine brakes have an important role in wind turbine technology. Therefore, the study of establishing dynamics model for the wind turbine brakes, simulating and analyzing the braking process through different computer-aided analysis software has an important significance. In this paper, a 3D model of the wind turbine brakes and a rigid-flexible-coupling model are established. By simulating and analyzing the dynamic performance, the braking process of typical conditions are studied considering the friction coefficient, braking power and flexible members.

\section{The Theoretical Model of Wind Turbine Brakes}

Working Principle. There are 3 key parts in a wind turbine brake, brake caliper, brake disc and brake pad. When the wind turbines work normally, the oil under pressure gets into the brake cylinder through main oil line and push the piston back in order to maintain a certain gap between the brake disc and brake pad. When wind turbines require an emergency stop, the oil under pressure flows back into the oil tank through the offloading loop as the power of motor and electromagnetic value cut off, then compression springs push forward the piston, which makes a contact between the brake pad and the friction disc to apply the brake.

Motion Equations of the Brakes' Flexible Body. Since flexible bodies have unlimited DOF (degree of freedom), it is essential to reduce the DOF in order to ensure sufficient accuracy. The original modes are replaced by a new mode made up with the reduced primary modes and constraints modes. The deformation of nodes $u$ is defined in formula (1):

$$
u=\sum \phi_{i} q_{i} \quad(i=1 \sim m)
$$

Where, $m$ is the total number flexible body mode; $i$ is the ordinal number of flexible body mode; $q_{i}$ is the coordinate of flexible body mode.

Generalized coordinate is defined as, $\xi=(X, \psi, q)$, where, $X=(x, y, z)$ is Cartesian coordinate in the overall reference system; $\psi=(\psi, \theta, \varphi)$ is Euler angle reflecting the rigid position; $q=\left\{q_{1}, q_{2}, \ldots\right.$, $\left.q_{m}\right\}^{T}$ is the modal coordinate. The equation of motion of the flexible body [3] written by the Lagrange multiplier method is defined as the formula (2): 


$$
\frac{d}{d t}\left[\frac{\partial L}{\partial \xi}\right]-\frac{\partial L}{\partial \xi}+\frac{\partial \theta}{\partial \xi}+\left[\frac{\partial \psi}{\partial \xi}\right]^{T} \lambda-Q=0
$$

Where, $L$ is Lagrangian function, $\Psi$ is constraint equation; $\lambda$ is Lagrange multiplier; $Q$ is generalized force.

\section{The Simulation Model of Wind Turbine Brakes}

The Definition of Motion Constraints in Simulation Model. After assembling the components, virtual prototype model is established by importing format file PARASOLID interface model into ADAMS [3], as shown in Fig.1. After assembling the wind turbine brake's model in ADAMS/View, motion constraints relationship between the components is established according to the actual situation of the brake (as shown in Tab.1). Contact friction is established between the brake pad and brake disc and the contact parameters are shown in Tab.2. The simulation model of wind turbine brake is shown in Fig.2.

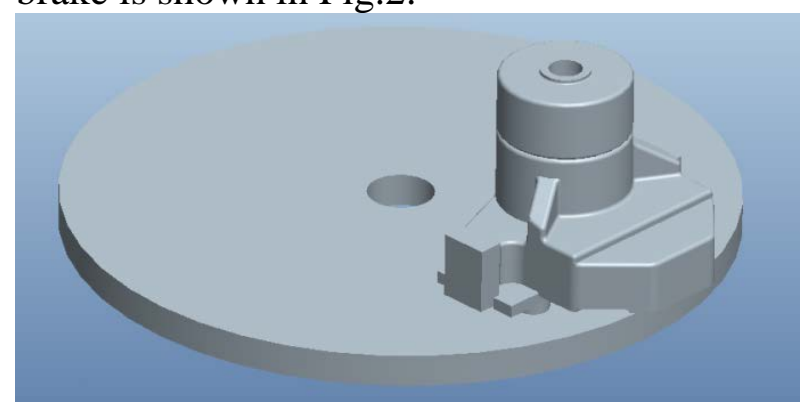

Fig.1. 3D model of wind turbine brakes

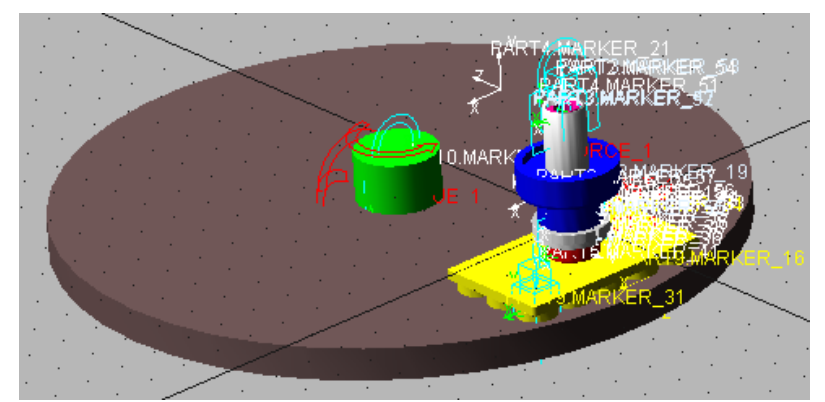

Fig.2. Simulation model of wind turbine brakes

Tab.1. the constraint relationships between components of brake

\begin{tabular}{cc}
\hline Components & Joint \\
\hline brake disc and ground & Revolute \\
brake pads and ground & Translational \\
outer sleeve and brake pads & Fixed \\
nut and outer sleeve & Translational \\
screw and nut & Translational \\
screw and sleeve & Fixed \\
nut and guide bar & Fixed \\
\hline
\end{tabular}

Tab.2. Contact parameters of brake disc and brake pads

\begin{tabular}{cccccccc}
\hline $\begin{array}{c}\text { Stiffness } \\
\mathrm{K} /\left(\mathrm{N} \cdot \mathrm{mm}^{-1}\right)\end{array}$ & $\begin{array}{c}\text { Damping } \\
\mathrm{C} /(\mathrm{N} \cdot \mathrm{s} \cdot \mathrm{m} \\
\left.\mathrm{m}^{-1}\right)\end{array}$ & $\begin{array}{c}\text { Nonlinear } \\
\text { index e }\end{array}$ & $\begin{array}{c}\text { Deformati } \\
\text { on depth } \\
\mathrm{D} / \mathrm{mm}\end{array}$ & $\begin{array}{c}\text { Static } \\
\text { coefficient }\end{array}$ & $\begin{array}{c}\text { Dynamic } \\
\text { factor }\end{array}$ & $\begin{array}{c}\text { Static slip } \\
\text { velocity } \\
\mathrm{vs} /\left(\mathrm{mm} \cdot \mathrm{s}^{-1}\right)\end{array}$ & $\begin{array}{c}\text { Slip rate } \\
\mathrm{vs} /\left(\mathrm{mm} \cdot \mathrm{s}^{-1}\right)\end{array}$ \\
\hline $2.1 \times 10^{5}$ & 10.0 & 2.2 & 0.1 & 0.4 & 0.1 & 100 & 1000 \\
\hline
\end{tabular}

The Settings of Drivers and Loads. The simulation is referring to the brake process in 1000rpm working conditions: Firstly, add a starting torque at the beginning of the process, after 5 s remove the torque, the brake disc maintains 1000rpm uniform motion. The starting torque is set by STEP function [4] [5], as follows:

STEP( time ,0 0, 0.1, 4091500)+STEP( time , 0.1, 0, 5.1,0 )

+ STEP( time, 5.1, 0, 5.2,-4091500)

During the brake process, firstly, add a custom constant power in the eighth second, braking torque is generated by friction ans the braking process is complete when the brake disc stopped completely. The function of the braking force is following:

STEP( time , $0,0,8,0$ )+STEP( time , 8,0,10,-17000) 


\section{Rigid-coupling Dynamic Characteristics Analysis of Wind Turbine Brakes}

The Influence of the Friction Coefficient in Braking Process. After applying the braking force in 8 s, the brake brakes under the effect of the friction torque. The simulation curves are shown as Fig.3 in braking process, when the friction coefficient of 0.3(solid line) and 0.4(dash line).

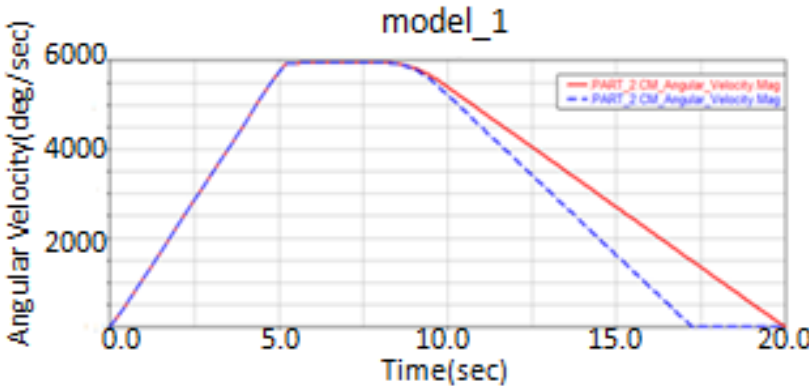

(a) Angular velocity curves

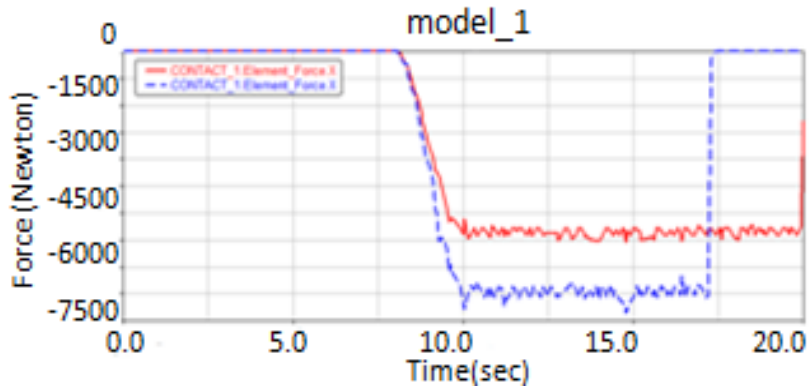

(b) Contact force curves

Fig.3. The experimental results in different friction coefficient

According to the different friction coefficient of braking curves (angular velocity curve), the two curves coincided in $0 \sim 8 \mathrm{~s}$, which proved that the acceleration and constant speed process in both simulation procedure are the same. After 8 s the brake started to brake at the same time, and stopped when the angular velocity is zero. The greater the friction coefficient is, the lower angular velocity reduces, the shorter braking time is and the better braking performance we can obtain. The conclusions that extent of vibration increases with the friction coefficient can also be proved. When selecting the material of the brake pad with appropriate coefficient of friction, ensuring braking performance and suppressing the vibration of the brake are required at the same time.

The Influence of the Braking Force in Braking Process. The simulation curves are shown as Fig.4. in braking process, when the braking force is $10000 \mathrm{~N}$ (solid line), $15000 \mathrm{~N}$ (dash line) and $20000 \mathrm{~N}$ (dot line).

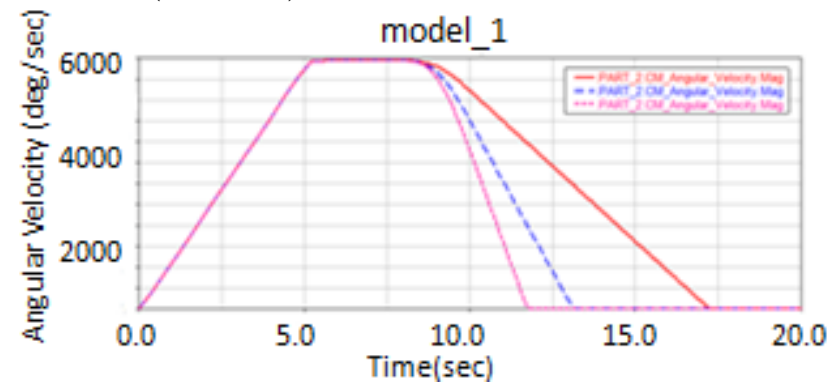

(a) Angular velocity curves

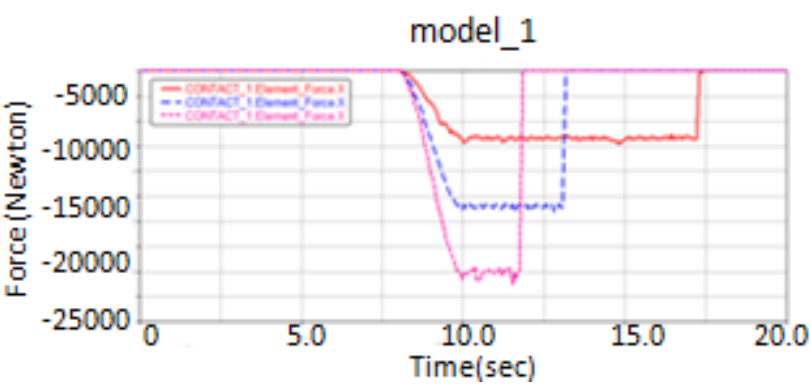

(b) Contact force curves

Fig.4. The experimental results in different braking force

According to comparison of the brake curves, similar to the coefficient of friction, the slope of the curves also present regular changes in different braking force. Braking performance increased as the braking force was increasing, and the braking time reduced at the same time. According to the contact force curves, the contact friction is increasing with the braking force, and the volatility of friction is increasing with the braking force. The larger magnitude of friction of brake disc may cause vibration, which may affect the braking process. Therefore, when selecting the braking force, braking time and the degree of fluctuation of the friction are both taken into account. The stiffness of each component of the brake is also considered in operation.

The Influence of the Rotational Speed in Braking Process. The simulation curves are shown as Fig.5 in braking process, when the rotational speed is 500rpm(solid line), 1000rpm(dash line) and 2000rpm(dot line). 


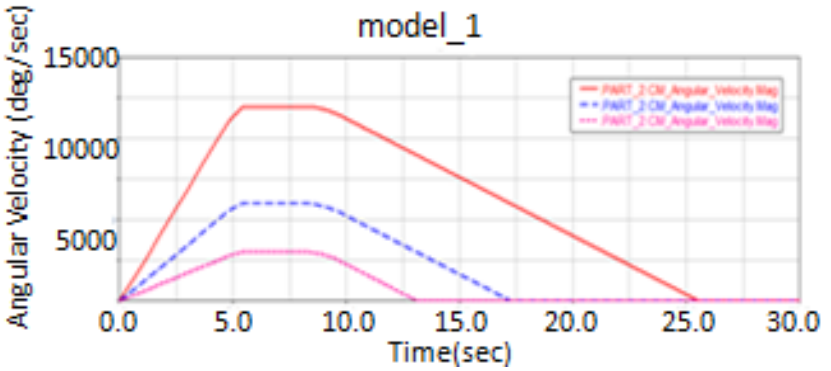

(a) Angular velocity curves

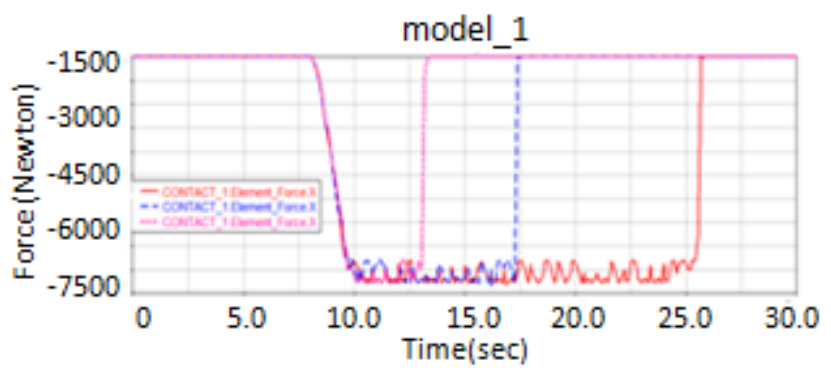

(b) Contact force curves

Fig.5. The experimental results in different rotational speed

According to the brake curves, the curves have the same slope after 8s, which proved that all the factors are the same except rotational speed. According to the contact force curves, due to the friction factor and the contact forces are the same, the maximum amplitude of contact friction in different conditions is similar. But in the condition of 500rpm, only few vibration reached to the maximum amplitude, others were significantly less than the maximum amplitude; in the condition of 1000rpm, approximately one-third of vibration reached to the maximum amplitude, others were slightly higher than the magnitude of 500rpm; in the condition of 2000rpm, amplitude were all closed to the maximum amplitude. The higher the speed is, the more unstable the vibration in braking process is. Therefore, it is necessary to consider the impact of vibration in the braking process according to different conditions.

\section{Conclusion}

A virtual prototype of megawatt wind turbine brake is established, and the braking process of megawatt wind turbine brake is simulated in typical operating conditions. A dynamic characteristic analysis is applied for the model of megawatt wind turbine brake adding the driving torque and different loads. Considering the influence of the friction coefficient and braking force in braking time and contact force, the dynamics simulation results of wind brake process are obtained. This approach provides the model reference for structural design and optimization of the brake by the visualizing simulation process.

\section{Acknowledgement}

In this paper, the research was sponsored by the National Natural Science Foundation of China (Grant No. 51475066) and Dalian Municipal Science and Technology Foundation (Grant No. 2013J21DW011).

\section{References}

[1] Xue Heng, Zhu Ruizhao, Yang Zhenbin, etc. ASSESSMENT OF WIND ENERGY RESERVES IN CHINA[J]. Acta Energiae Solaris Sinica, 2001, 22(2): 167-170.

[2] Fang Chuanglin. The Analysis and Prospect of Wind Power Development Goals of China[J]. Energy of China, 2007, 12(29): 30-34.

[3] Ning Xiaobin, Zhang Wenming. Multi-body Dynamic Simulation for Analysis of Disc Brake Vibration. Nonferrous Metals, 2004 56(4): 119-12.

[4] Chen Demin, Huai Chuangfeng, Zhang Ketao, etc, ADAMS 2005/2007 proficient virtual prototyping technology[M], Beijing: Chemical Industry Press, 2010.

[5] Chen Liping, Zhang Shiqing, Ren Weiqun, etc, Mechanical System Dynamics Analysis and Application Tutorials of ADAMS [M], Beijing: Tsinghua University Press, 2005. 